\title{
Active Fault Tolerant Control Based on Bond Graph Approach
}

\author{
Manel Allous ${ }^{1}$ and Nadia Zanzouri ${ }^{1,2}$ \\ ${ }^{1}$ Department of Electrical Engineering, LACS Laboratory, National Engineering School of Tunis, \\ Université de Tunis El Manar, P.O. Box 37, Le Belvedere, 1002 Tunis, Tunisia \\ ${ }^{2}$ Preparatory Engineering Institute of Tunis 2, Université de Tunis, Rue Jawaher Lel Nahrou-Monfleury, 1089 Tunis, Tunisia \\ Correspondence should be addressed to Manel Allous; allousmanel@yahoo.fr
}

Received 30 April 2014; Revised 21 September 2014; Accepted 21 September 2014; Published 10 November 2014

Academic Editor: Gorazd Stumberger

Copyright (C) 2014 M. Allous and N. Zanzouri. This is an open access article distributed under the Creative Commons Attribution License, which permits unrestricted use, distribution, and reproduction in any medium, provided the original work is properly cited.

This paper proposes a structural fault recoverability analysis using the bond graph (BG) approach. Indeed, this tool enables structural analysis for diagnosis and fault tolerant control (FTC). For the FTC, we propose an approach based on the inverse control using the inverse BG. The fault tolerant control method is also compared with another approach. Finally, simulation results are presented to show the performance of the proposed approach.

\section{Introduction}

Due to the growing complexity of the dynamical systems, there is an increasing demand for safe operation, fault diagnosis (FDI) (fault detection and isolation), and fault tolerant control (FTC) (strategies for control redesign). Different approaches have been developed for the designing and the implementation of FDI and FTC procedures [1]. These techniques are based on the knowledge of the system model (model-based methods) $[2,3]$ or its structure (databased methods) $[4,5]$. FTC is categorized into two different techniques: passive FTC (PFTC) [6,7] and active FTC (AFTC) $[8,9]$. In PFTC, controllers are fixed and designed to be robust against a class of presumed faults. The AFTC approach reacts to system component failures actively by reconfiguring control actions and acceptable performance of the entire system can be maintained.

This paper is focused on the design of a novel AFTC that integrates a reliable and robust fault diagnosis scheme with the design of a controller reconfiguration system. The FDI and FTC are fully integrated in dynamic systems design in several fields of engineering, such as robotic and automotive systems. Nevertheless, it must have tool that enables coupling the diagnosis results with fault tolerant control conditions. Therefore, the BG enables integrating both structural diagnosis results with control analysis. A BG model allows knowledge of a large amount of structural, functional, and behavioral information. This information enables computing appropriate control actions that compensate the faults.

The BG has proven to be a powerful tool not only for generating the direct model of a system but also for obtaining its inverse model. In [10], the authors have proposed an inverse control strategy based on BG model.

The innovative interest of the present paper is to combine the inverse control strategy and observer designs to generate the FDI and FTC algorithms from the BG model. The proposed approach takes into account the parameter uncertainties and considers the fault recoverability with respect to fault compensation, without complex calculations.

In the first part of the paper, we propose a methodology based on BG model for fault detection and fault tolerant control. In the second part, we have developed a method which compensates the faults in the absence of complex calculations. Finally, an illustrative example is developed and simulation results show the advantage of the proposed approach.

\section{FDI and FTC Approaches Based on Bond Graph}

The bond graph approach is proposed by [11] and then developed by $[12,13]$. This tool allows the multidomain 


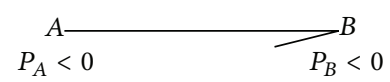

Figure 1: Power and orientation symbol on BG.

TABLE 1: BG elements.

\begin{tabular}{lc}
$\stackrel{e}{\vec{f}} R$ & $S_{e} \underset{f}{\stackrel{e}{f}} ; S_{f} \frac{e}{f}$ \\
$\stackrel{e=d p / d t}{\vec{f}} I$ & $\frac{e_{1}}{f_{1}} \mathrm{~Gy} \frac{e_{2}}{f_{2}}$ \\
$\stackrel{e}{f=d q / d t} \mathrm{e}$ & $\frac{e_{1}}{f_{1}} \mathrm{TF} \frac{e_{2}}{f_{2}}$ \\
\hline
\end{tabular}

systems (mechanical, electrical, thermal, etc.) to be described with the same components. Its causal structure was initially exploited to determine structural conditions of controllability, observability [14], and diagnosability $[15,16]$.

The bond graph is based on the graphical representation of the energy exchange within the system to be modeled. Table 1 represents the BG elements: resistor $(R)$, compliance $(C)$, and inertia $(I)$ are passive elements. Effort source $\left(S_{e}\right)$ and flow source $\left(S_{f}\right)$ are the active elements.

Figure 1 indicates the power direction in the system.

There are only two types of junctions: the 1 and the 0 junctions (Figure 2). 1 junction has equality of flows and the efforts sum up to zero. 0 junctions have equality of efforts and the flows sum up to zero (Figure 2).

2.1. Luenberger Observer Based on BG. Bond graph approaches for observers design were developed in some works, such as Luenberger observers $[17,18]$, reduced order Luenberger observers [19], proportional integral observers [20], and nonlinear observers applied to electrical transformers [21].

The objective of this work is to design a Luenberger observer by bond graph for fault detection and isolation.

2.2. BG Modeling Bicausality Concept for System Inversion. The concept of "bicausal" introduced by Gawthrop [22] enlarges the possibilities of computation models that can be derived from a bond graph. The bicausal bond graph model is seen as half-strokes each associated with an effort and a flow variable that can be imposed independently at each end of the bond. Causal half-strokes indicate the fixed or known variables of the bond and so determine the right-hand side of the assignments form [23] (Figure 3).

The bicausality is used to get systems' inversion by imposing the output variable without modifying the energy structure. System inversion is an interesting analysis to know an input considering a given output. Therefore, in the next section, we use the bicausality property of the bond graphs.

Some conditions (structural invertibility) are proposed to present the bond graph-based procedure for system inversion [20].

Proposition 1. A linear system modeled by bond graph is invertible if there is at least one causal path between the input variable and the output variable of the system.

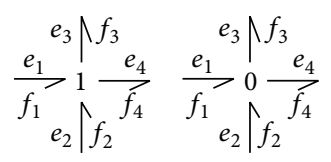

FIGURE 2: BG junctions.

$$
\begin{array}{cc}
\text { System } 1 \underset{f_{1} \longleftarrow f_{2}}{\stackrel{e_{1}}{\longleftarrow} \text { System 2 }} & \text { System 1 } e_{2} e_{1} \longrightarrow e_{2} \text { System 2 } \\
\left\{\begin{array}{l}
e_{1}=e_{2} \\
f_{1}=f_{2}
\end{array}\right. & \left\{\begin{array}{l}
e_{2}=e_{1} \\
f_{2}=f_{1}
\end{array}\right.
\end{array}
$$

FIGURE 3: Bicausal bond graph concept.

2.3. Control Law Design Directly on Inversion Bond Graph. The control strategy proposed by [24] computes the desired inputs based on the system objectives. Also, in [10], the authors have proposed an inverse control strategy from the BG model with parameter uncertainties estimated directly from the inverse BG-LFT (linear fractional transformation). The system inversion concept gives the basis to compute appropriate control actions that compensate the faults. Figure 4 shows the control design based on bond graph developed by [10].

In [10], the BG-LFT was used to estimate the faulty power. Then, to validate these structural results, a local adaptive compensation based on the inverse control strategy using the inverse BG-LFT was proposed. This strategy computes the desired inputs based on the system objectives and on the undesired power caused by the fault.

Limitations of this approach are as follows.

(i) The fault estimation is necessary for the control design. The inverted model of bond graph uses the estimate fault to compensate it.

(ii) The fault estimation with a BG-LFT causes FTC delay.

\section{Proposed Approach}

The principal of our proposed approach AFTC system is presented in Figure 5. There are basically two parts.

(i) The first part concerns the diagnosis by Luenberger observer using BG approach; in this part, the fault estimation and fault isolability are not necessary for system recoverability; just the residual is injected to the control loop.

(ii) The second part shows the control part determined by inverse BG for nominal system.

The following symbols have been used.

I.BG.N.S: Inverse BG for nominal system.

$y_{\text {ref: }}$ Desired value.

$y_{\text {sys }}:$ Measured output of system.

$y_{\text {obs }}$ : Estimated output of observer. 


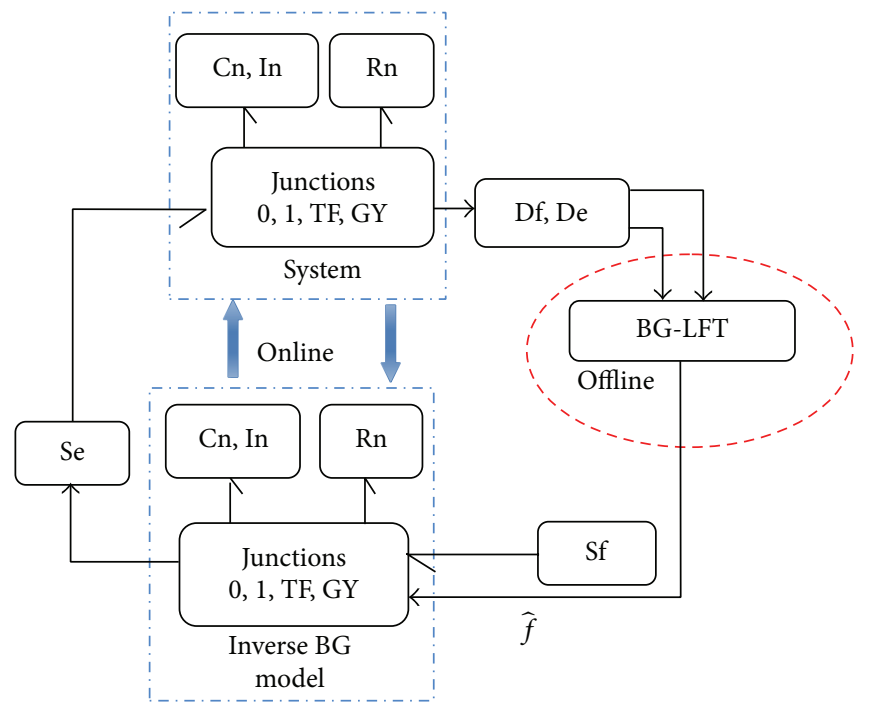

FIGURE 4: Control design on bond graph developed by [10].

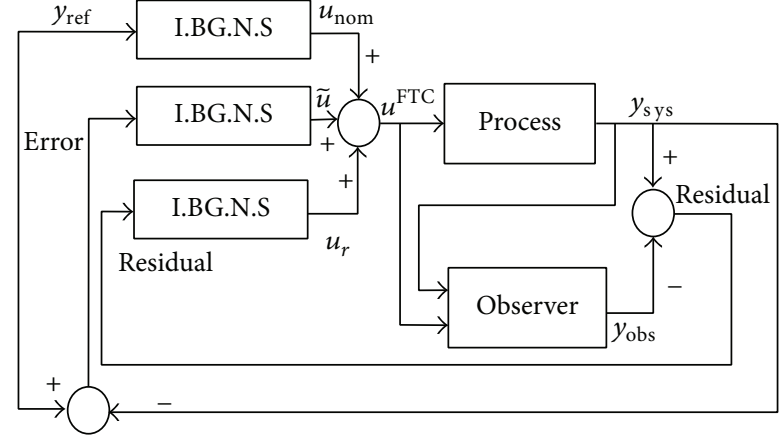

FIGURE 5: AFTC strategy based on bond graph.

Computing the Control $\left(u^{F T C}\right)$. Various methods have been proposed to recover as close as possible the system performance according to the considered fault representation.

Some extensions of the classical pseudoinverse method (PIM) have been proposed to guarantee both the performance and the stability of the faulty system. The authors in $[25,26]$ have synthesized a suitable feedback control $K_{\text {feedback }}$. In $[27,28]$, the authors have proposed to compute a reconfigurable forward gain $K_{\text {forward }}$ controller in order to eliminate the steady-state tracking error in faulty case. Therefore, the control signal applied to the system is represented in

$$
u^{\mathrm{FTC}}=K_{\text {forward }} y_{\text {ref }}-K_{\text {feedback }} x .
$$

A novel technique to adjust the command equation (1) is proposed by [29] in

$$
u^{\mathrm{FTC}}=K_{\text {forward }} y_{\text {ref }}+\tilde{u} \text {. }
$$

$\tilde{u}$ given by

$$
\tilde{u}=K_{\text {feedback }}\left(x-x^{n}\right)
$$

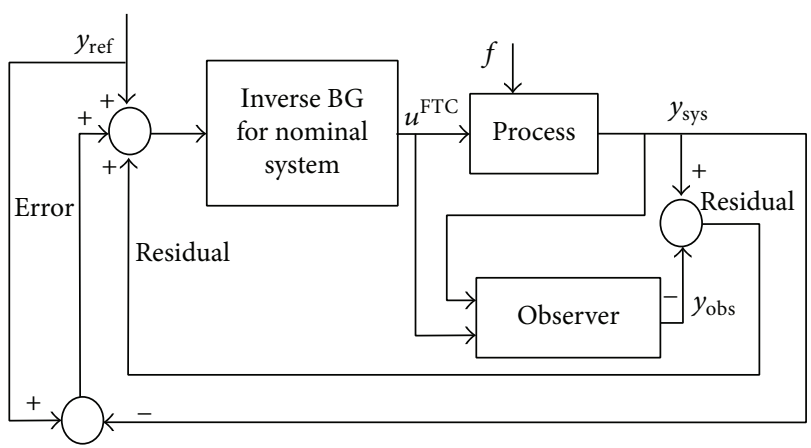

Figure 6: New control based on bond graph.

According to the control law in (1) and (2), we propose a new control which uses the residual signal provided by the FDI based observer and error. So, the control law is expressed by

$$
\begin{aligned}
u^{\mathrm{FTC}}= & K_{\text {forward }} y_{\text {ref }}+K_{\text {forward }}(\underbrace{y_{\text {ref }}-y_{\text {sys }}}_{\text {error }}) \\
& +K_{\text {forward }}(\underbrace{y_{\text {sys }}-y_{\text {obs }}}_{\text {residual }}) .
\end{aligned}
$$

Or $K_{\text {forward }}$ is a gain of inverse BG for nominal system.

In Figure 5, the compensation term $u_{r}$ (residual control) is useful to compensate the fault. Also $\widetilde{u}$ (error control) is added to the nominal control $\left(u_{\text {nom }}\right)$; this term $(\widetilde{u})$ improves the compensation of the fault effect. So this additive control results' role is to reproduce the control signal $\left(u^{\mathrm{FTC}}\right.$ : controlled input resulting from (4) for compensating for the effect of the fault every moment that the fault is detected).

To simplify the calculus of the control input represented in Figure 5, we propose to replace the three inverse BG models by a single inverse BG (Figure 6). 
TABLE 2: Parameter values of the DC motor.

\begin{tabular}{lc}
\hline$R=1 \Omega$ & Rotor resistance \\
$L=5 \mathrm{mH}$ & Rotor inductance \\
$b=10^{-4} \mathrm{Nm} / \mathrm{rd} \cdot \mathrm{s}^{-1}$ & Coefficient of viscous \\
$J=0.001 \mathrm{Kg} \mathrm{m}$ & Moment of inertia \\
$k_{n}=0.2 \mathrm{Nm} / \mathrm{A}$ & Coefficient of the torque \\
$u_{\text {nom }}=220 \mathrm{~V}$ & Motor voltage \\
\hline
\end{tabular}

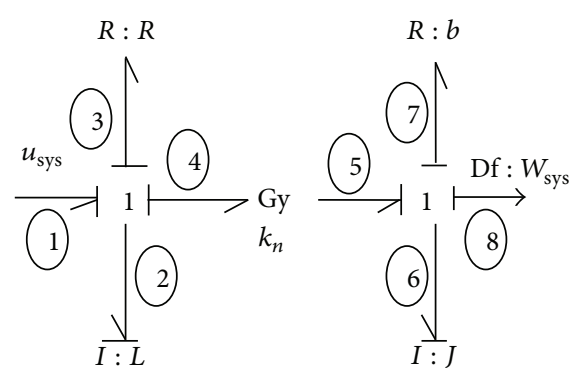

Figure 7: Bond graph model of DC motor.

So, the new control law is expressed by

$$
u^{\mathrm{FTC}}=K_{\text {forward }}\left(y_{\text {ref }}+\text { error }+ \text { residual }\right) .
$$

\section{Illustrative Example}

An example of a DC motor is used to illustrate our new FTC technique. The BG model of the system is given in Figure 7, and the state-space equations are presented in (6);

$$
\begin{gathered}
{\left[\begin{array}{l}
\dot{x}_{1} \\
\dot{x}_{2}
\end{array}\right]=\left[\begin{array}{cc}
-\frac{b}{J} & \frac{k_{n}}{J} \\
-\frac{k_{n}}{L} & -\frac{R}{L}
\end{array}\right]\left[\begin{array}{l}
x_{1} \\
x_{2}
\end{array}\right]+\left[\begin{array}{l}
0 \\
\frac{1}{L}
\end{array}\right] u,} \\
y=\left[\begin{array}{ll}
1 & 0 \\
0 & 1
\end{array}\right]\left[\begin{array}{l}
x_{1} \\
x_{2}
\end{array}\right]+\left[\begin{array}{c}
0.1 \\
0
\end{array}\right] d,
\end{gathered}
$$

with $x=\left(x_{1}, x_{2}\right)=(w, i)$ being the state vector, $y$ the measured output variable, $u$ the control input variable, and $d$ the disturbance input variable.

The parameters of the DC motor are presented in Table 2.

Closed Loop System. Figure 8 shows that the AFTC strategy integrates the FDI module with an inverse BG for nominal system and the bond graph model is controllable and observable [14].

From the controlled input and output signals, the FDI module provides the residual which is injected to the control loop, in order to compensate the effect of fault.

The objective is to synthesize a controller so that the structure of the closed loop system is as close as possible to that of the desired reference model under the normal operation or in the presence of fault.

(i) Computing the Residual ( $r$ ) in Normal Operating. By causal path, we deduce the structural equations from the BG of Figure 8. We compute the residual $r_{1}$ with the following equations: (a) structural equations for system model:

$$
\begin{aligned}
& f_{1_{s}}=f_{2_{s}}=f_{3_{s}}=f_{4_{s}}, \\
& e_{1_{s}}=e_{2_{s}}+e_{3_{s}}+e_{4_{s}} ; \\
& e_{4_{s}}=k_{n} f_{5_{s}}, \\
& e_{5_{s}}=k_{n} f_{4_{s}} ; \\
& f_{5_{s}}=f_{6_{s}}=f_{7_{s}}=f_{8_{s}}, \\
& e_{5_{s}}=e_{6_{s}}+e_{7_{s}}+e_{8_{s}} ; \\
& f_{5_{s}}=\frac{e_{4_{s}}}{k_{n}}, \\
& f_{5_{s}}=\frac{e_{1_{s}}-e_{2_{s}}-e_{3_{s}}}{k_{n}} ;
\end{aligned}
$$

(b) structural equations for Luenberger observer:

$$
\begin{aligned}
& f_{1_{\mathrm{ob}}}=f_{2_{\mathrm{ob}}}=f_{3_{\mathrm{ob}}}=f_{4_{\mathrm{ob}}}, \\
& e_{1_{\mathrm{ob}}}=e_{2_{\mathrm{ob}}}+e_{3_{\mathrm{ob}}}+e_{4_{\mathrm{ob}}} ; \\
& e_{4_{\mathrm{ob}}}=k_{n} f_{5_{\mathrm{ob}}}, \\
& e_{5_{\mathrm{ob}}}=k_{n} f_{4_{\mathrm{ob}}} ; \\
& f_{5_{\mathrm{ob}}}=f_{6_{\mathrm{ob}}}=f_{7_{\mathrm{ob}}}=f_{8_{\mathrm{ob}}}, \\
& e_{5_{\mathrm{ob}}}=e_{6_{\mathrm{ob}}}+e_{7_{\mathrm{ob}}}+e_{8_{\mathrm{ob}}} ; \\
& f_{5_{\mathrm{ob}}}=\frac{e_{4_{\mathrm{ob}}}}{k_{n}}, \\
& f_{5_{\mathrm{ob}}}=\frac{e_{1_{\mathrm{ob}}}-e_{2_{\mathrm{ob}}}-e_{3_{\mathrm{ob}}}}{k_{n}} .
\end{aligned}
$$

From junctions equations (7) and (8), we generate the residual $r_{1}$ :

$$
\begin{gathered}
r_{1}=f_{8_{s}}-f_{8_{\mathrm{ob}}}=f_{6_{s}}-f_{6_{\mathrm{ob}}}=f_{5_{s}}-f_{5_{\mathrm{ob}}}=\frac{e_{4_{s}}}{k_{n}}+\frac{e_{4_{\mathrm{ob}}}}{k_{n}}, \\
f_{5_{s}}=\frac{1}{k_{n}}\left[U_{s}-L \frac{d f_{2_{s}}}{d t}-R f_{2_{s}}\right], \\
f_{5_{\mathrm{ob}}}=\frac{1}{k_{n}}\left[U_{\mathrm{ob}}-L \frac{d f_{2_{\mathrm{obs}}}}{d t}-R f_{2_{\mathrm{ob}}}-r_{1} K_{11}\right], \\
r_{1}=\frac{1}{k_{n}}\left[U_{s}-L \frac{d f_{2_{s}}}{d t}-R f_{2_{s}}-U_{\mathrm{ob}}+L \frac{d f_{2_{\mathrm{ob}}}}{d t}+R f_{2_{\mathrm{ob}}}\right] .
\end{gathered}
$$




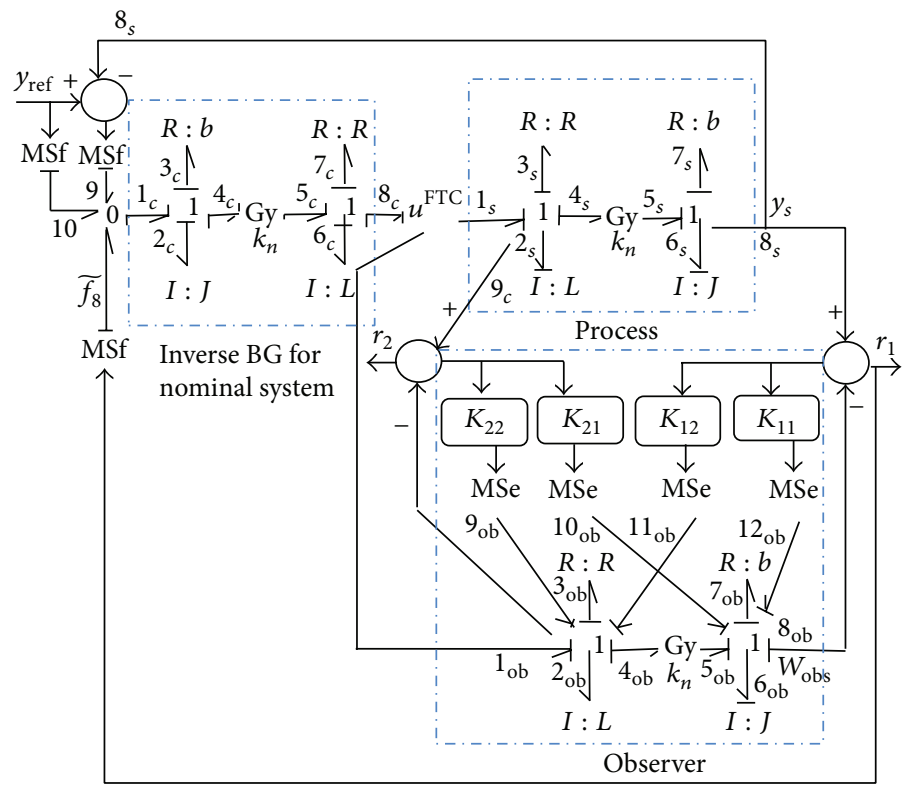

Figure 8: FTC based on BG model.

$\mathrm{Or}$

$$
\begin{aligned}
& U_{s}=U_{\mathrm{obs}} \text {, } \\
& f_{2_{s}}=\frac{e_{5_{s}}}{k_{n}}=\frac{1}{k_{n}}\left[J \frac{d f_{6_{s}}}{d t}+b f_{6_{s}}\right] \text {, } \\
& f_{2_{\mathrm{ob}}}=\frac{e_{5_{\mathrm{ob}}}}{k_{n}}=\frac{1}{k_{n}}\left[J \frac{d f_{6_{\mathrm{ob}}}}{d t}+b f_{6_{s}}-K_{11} r_{1}\right] \text {, } \\
& r_{1}=\frac{1}{k_{n}}\left[U_{s}-L \frac{d\left(1 / k_{n}\right)\left[J\left(d f_{6_{s}} / d t\right)+b f_{6_{s}}\right]_{2}}{d t}\right. \\
& -R \frac{1}{k_{n}}\left[J \frac{d f_{6_{s}}}{d t}+b f_{6_{s}}\right]-U_{\mathrm{ob}} \\
& +L \frac{d\left(1 / k_{n}\right)\left[J\left(d f_{6_{\mathrm{ob}}} / d t\right)+b f_{6_{s}}-K_{11} r_{1}\right]}{d t} R \\
& \left.\times \frac{1}{k_{n}}\left[J \frac{d f_{6_{\mathrm{ob}}}}{d t}+b f_{6_{s}}-K_{11} r_{1}\right]-r_{1} K_{12}\right] .
\end{aligned}
$$

The residual $r_{1}$ of the system is realized as

$$
\begin{aligned}
r_{1}= & \frac{-J L}{k_{n}^{2}} \frac{d^{2} r_{1}}{d t}-\frac{d r_{1}}{d t}\left[\frac{R J+L b+K_{11} L}{k_{n}^{2}}\right] \\
& -r_{1}\left[\frac{R b+K_{11} R}{k_{n}^{2}}+\frac{K_{12}}{k_{n}}\right],
\end{aligned}
$$

$$
\left[1+\frac{R b+K_{11} R}{k_{n}^{2}}+\frac{K_{12}}{k_{n}}\right] r_{1}+\left[\frac{R J+L b+K_{11} L}{k_{n}^{2}}\right] \frac{d r_{1}}{d t}
$$$$
+\frac{J L}{k_{n}^{2}} \frac{d^{2} r_{1}}{d t}=0
$$

The $r_{2}$ is deduced with similar method.

The inverse system enables computing appropriate control actions that compensate the faults.

(ii) Computing the Control $\left(u^{F T C}\right)$. The control law can be designed directly from the BG model:

structural equations for inverse BG model:

$$
\begin{aligned}
& f_{1_{c}}=f_{2_{c}}=f_{3_{c}}=f_{4_{c}}, \\
& e_{1_{c}}=e_{2_{c}}+e_{3_{s}}+e_{4_{s}} ; \\
& e_{4_{c}}=k_{n} f_{5_{c}}, \\
& e_{5_{c}}=k_{n} f_{4_{c}} ; \\
& f_{5_{c}}=f_{6_{c}}=f_{7_{c}}=f_{8_{c}}, \\
& e_{5_{c}}=e_{6_{c}}+e_{7_{c}}+e_{8_{c}} .
\end{aligned}
$$




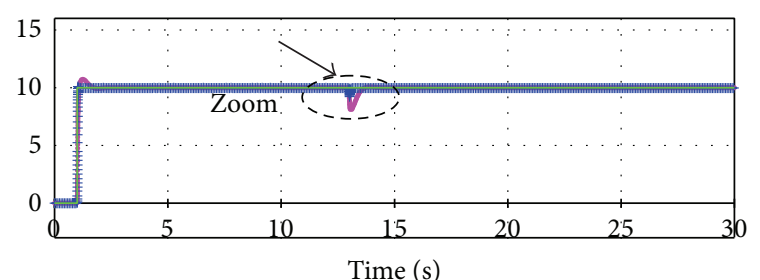

- Velocity (approach of [10])

+ Velocity (our approach)

— Desired velocity

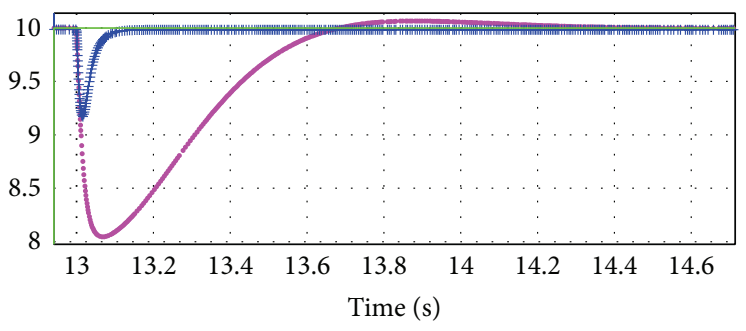

- Velocity (approach of [10])

+ Velocity (our approach)

— Desired velocity

FIGURE 9: Simulation results when the fault is compensated.

The control law $u^{\mathrm{FTC}}$ of the system is shown as

$$
\begin{gathered}
u^{\mathrm{FTC}}=e_{8_{c}}=e_{5_{c}}+e_{6_{c}}+e_{7_{c}}, \\
e_{5_{c}}=k_{n} f_{1_{c}}, \\
f_{1_{c}}=f_{10}+\tilde{f}_{8}+f_{9}, \\
f_{10}=w_{\mathrm{des}}=y_{\text {ref }}, \quad \tilde{f}_{8}=f_{8_{s}}-f_{8_{\mathrm{ob}}}=r_{1}, \\
f_{9}=w_{\mathrm{des}}-f_{8_{s}}=\varepsilon, \\
e_{6_{c}}=\frac{L d f_{6_{c}}}{d t}, \quad e_{7_{c}}=R f_{7_{c}}=R f_{6_{c}} .
\end{gathered}
$$

Or $r_{1}=$ residual and $\varepsilon=$ error.

So, the control law $u^{\mathrm{FTC}}$ is

$$
u^{\mathrm{FTC}}=R f_{6_{c}}+\frac{L d f_{6_{c}}}{d t}+k_{n}\left(\text { residual }+y_{\text {ref }}+\text { error }\right) \text {. }
$$

\section{Simulation Results}

Simulation results are carried out in the bond graph simulation software 20-sim [30] with parameter values described in Table 2.

Figure 9 shows the system output (velocity) evolution with a single fault (parameters $R: b, R_{\text {fault }}=R+\delta$, and $\delta=0.01)$ introduced at the time $13 \mathrm{~s}$.

We remark that the output decreases less than in the case of control considered in [10], and then it reaches the nominal values quicker at instant $t=13.05 \mathrm{~s}$. So, the control law (FTC) is able to stabilize the system on the desired output and to compensate the fault in the system with a very short time delay.

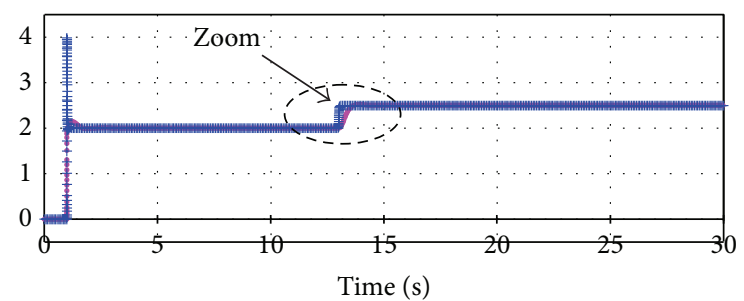

- U_FTC (approach of [10])

+ U_FTC (our approach)

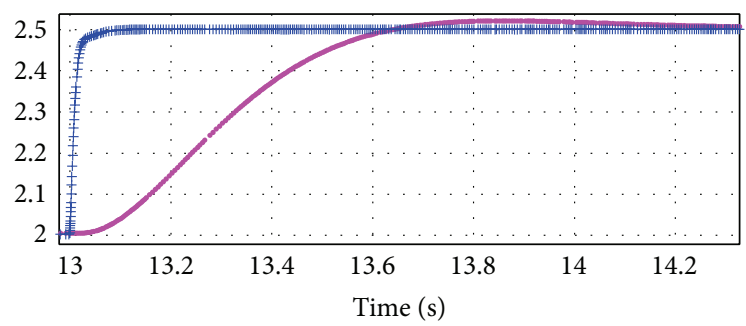

- U_FTC (approach of [10])

+ U_FTC (our approach)

Figure 10: Control input.

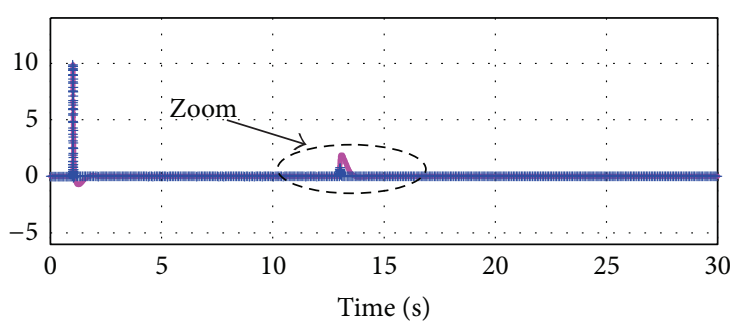

- Error of velocity (approach of [10])

+ Error of velocity (our approach)

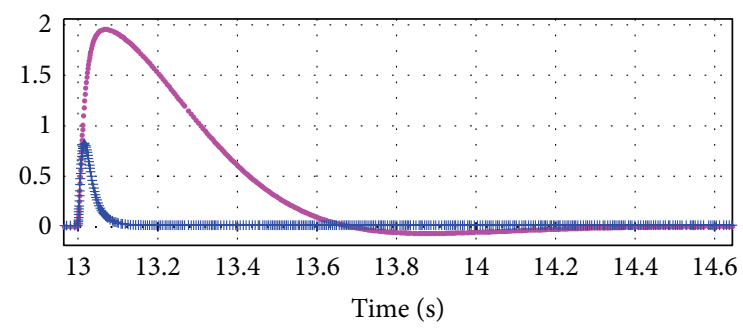

- Error of velocity (approach of [10])

+ Error of velocity (our approach)

FIGURE 11: Velocity error.

From a control point of view, the reconfigurable control mechanism requires more energy to reach the target and to guarantee system performance, as shown in Figure 10.

These results can be confirmed by the control input $U^{\text {FTC }}$ of Figure 10. In [10], the control input increases slowly trying to compensate for the fault affecting the system. In our approach, the control input increases quickly and enables rapid fault compensation on the controlled system output and allows compensating the convergence delay.

In Figure 11, the velocity error quickly converges to zero with the new approach. 


\section{Conclusion}

In this paper, we propose an active FTC design based on BG approach. The novel strategy combines an observer based model and inverse BG model.

The proposed approach enables computing appropriate control actions for compensating the faults. The faults are detected by Luenberger observer technique based on BG modeling. Fault isolation and fault estimation are not necessary to the FTC. The comparison between the two approaches shows the efficiency of the proposed method. The application of a FTC approach to induction DC motor and simulation results illustrate the performance of the proposed FDI-FTC structure. Our future works concern the online implementation of the proposed techniques on a real process.

\section{Conflict of Interests}

The authors declare that there is no conflict of interests regarding the publication of this paper.

\section{References}

[1] D. Chilin, J. Liu, X. Chen, and P. D. Christofides, "Fault detection and isolation and fault tolerant control of a catalytic alkylation of benzene process," Chemical Engineering Science, vol. 78, pp. 155-166, 2012.

[2] P. M. Frank, "Fault diagnosis in dynamic systems using analytical and knowledge-based redundancy. A survey and some new results," Automatica, vol. 26, no. 3, pp. 459-474, 1990.

[3] J. Gertler, "Fault detection and isolation using parity relations," Control Engineering Practice, vol. 5, no. 5, pp. 653-661, 1997.

[4] S. Srinivas, "A probabilistic approach to hierarchical modelbased diagnosis," in Proceedings of the 10th Conference on Uncertainty in Artificial Intelligence (UAI '94), pp. 538-545, 1994.

[5] Y. Qian, L. Xu, X. Li, L. Lin, and A. Kraslawski, "LUBRES: an expert system development and implementation for realtime fault diagnosis of a lubricating oil refining process," Expert Systems with Applications, vol. 35, no. 3, pp. 1252-1266, 2008.

[6] F. Liao, J. L. Wang, and G.-H. Yang, "Reliable robust flight tracking control: an LMI approach," IEEE Transactions on Control Systems Technology, vol. 10, no. 1, pp. 76-89, 2002.

[7] H. Niemann and J. Stoustrup, "Passive fault tolerant control of a double inverted pendulum-a case study," Control Engineering Practice, vol. 13, no. 8, pp. 1047-1059, 2005.

[8] P. Mhaskar, "Robust model predictive control design for faulttolerant control of process systems," Industrial and Engineering Chemistry Research, vol. 45, no. 25, pp. 8565-8574, 2006.

[9] Z. Zhang and W. Chen, "Adaptive output feedback control of nonlinear systems with actuator failures," Information Sciences, vol. 179, no. 24, pp. 4249-4260, 2009.

[10] R. Loureiro, R. Merzouki, and B. O. Bouamama, "Bond graph model based on structural diagnosability and recoverability analysis: Application to intelligent autonomous vehicles," IEEE Transactions on Vehicular Technology, vol. 61, no. 3, pp. 986-997, 2012.

[11] H. M. Paynter, Analysis and Design of Engineering Systems, The MIT Press, 1961.
[12] D. Karnopp, D. Margolis, and R. Rosenberg, Systems Dynamics: A Unified Approach, John Wiley and Sons, 1975.

[13] R. Rosenberg and D. C. Karnopp, Introduction to Physical System Dynamics, McGraw-Hill, 1983.

[14] C. Sueur and G. Dauphin-Tanguy, "Bond-graph approach for structural analysis of MIMO linear systems," Journal of the Franklin Institute, vol. 328, no. 1, pp. 55-70, 1991.

[15] B. Ould-Bouamama, A. K. Samantaray, M. Staroswiecki, and G. Dauphin-Tanguy, "Derivation of constraint relations from bond graph models for fault detection and isolation," in Proceedings of the International Conference on Bond Graph Modeling and Simulation (ICBGM '03), pp. 104-109, Orlando, Fla, USA, January 2003.

[16] Y. Touati, R. Merzouki, and B. Ould Bouamama, "Robust diagnosis to measurement uncertainties using bond graph approach: application to intelligent autonomous vehicle," Mechatronics, vol. 22, no. 8, pp. 1148-1160, 2012.

[17] D. Karnopp, "Bond graphs in control: physical state variables and observers," Journal of the Franklin Institute, vol. 308, no. 3, pp. 219-234, 1979.

[18] P. J. Gawthrop and L. P. S. Smith, Meta Modelling: Bond Graphs and Dynamic Systems, Prentice Hall, 1995.

[19] C. Pichardo-Almarza, A. Rahmani, G. Dauphin-Tanguy, and M. Delgado, "Bond graph approach to build reduced order observers in linear time invariant systems," in Proceedings of the 4th International Symposium on Mathematical Modelling, 2003.

[20] C. Pichardo-Almarza, A. Rahmani, G. Dauphin-Tanguy, and M. Delgado, "Proportional-integral observer for systems modelled by bond graphs," Simulation Modelling Practice and Theory, vol. 13, no. 3, pp. 179-211, 2005.

[21] G. Gonzalez-A and I. Nuñez, "A nonlinear observer of an electrical transformer: a bond graph approach," World Academy of Science, Engineering and Technology, vol. 58, pp. 814-820, 2009.

[22] P. J. Gawthrop, "Bicausal bond graphs," in Proceedings of the International Conference on Bond Graph Modelling and Simulation (ICBGM '95), pp. 83-88, 1995.

[23] R. F. Ngwompo, S. Scavarda, and D. Thomasset, "Inversion of linear time-invariant SISO systems modelled by bond graph," Journal of the Franklin Institute B: Engineering and Applied Mathematics, vol. 333, no. 2, pp. 157-174, 1996.

[24] N. Yadaiah and N. Venkata Ramana, "Linearisation of multimachine power system: modeling and control-a survey," International Journal of Electrical Power and Energy Systems, vol. 29, no. 4, pp. 297-311, 2007.

[25] Z. Gao and P. J. Antsaklis, "Stability of the pseudo-inverse method for reconfigurable control systems," International Journal of Control, vol. 53, no. 3, pp. 717-729, 1991.

[26] M. Staroswiecki, "Fault tolerant control: the pseudo-inverse method revisited," in Proceedings of the 16th Triennial World Congress of International Federation of Automatic Control (IFAC '05), pp. 418-423, July 2005.

[27] Y. M. Zhang and J. Jiang, "Active fault-tolerant control system against partial actuator failures," IEE Proceedings on Control Theory and Applications, vol. 149, no. 1, pp. 95-104, 2002.

[28] F. Guenab, D. Theilliol, P. Weber, J. C. Ponsart, and D. Sauter, "Fault tolerant control method based on cost and reliability analysis," in Proceedings of the 16th Triennial World Congress of International Federation of Automatic Control (IFAC '05), pp. 490-495, Prague, Czech Republic, July 2005. 
[29] Y. Zhang and J. Jiang, "Fault tolerant control system design with explicit consideration of performance degradation," IEEE Transactions on Aerospace and Electronic Systems, vol. 39, no. 3, pp. 838-848, 2003.

[30] 20 Sim Controllab Products B.V., http://www.20sim.com/. 

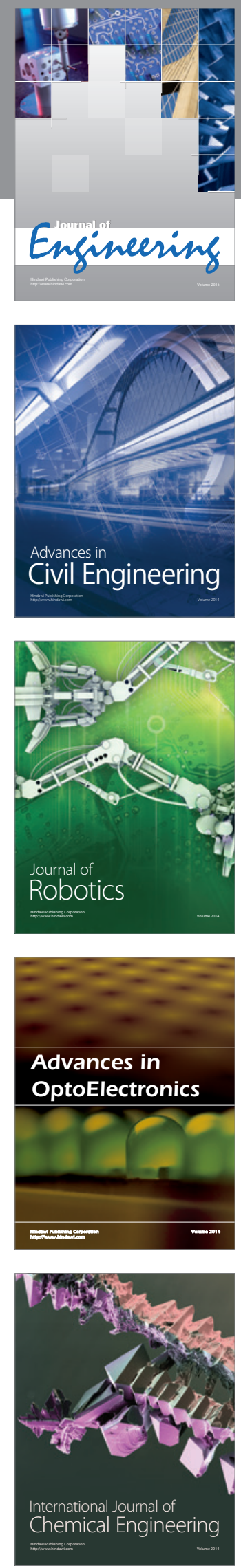

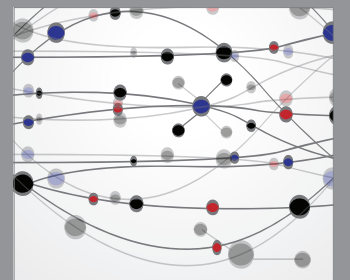

The Scientific World Journal
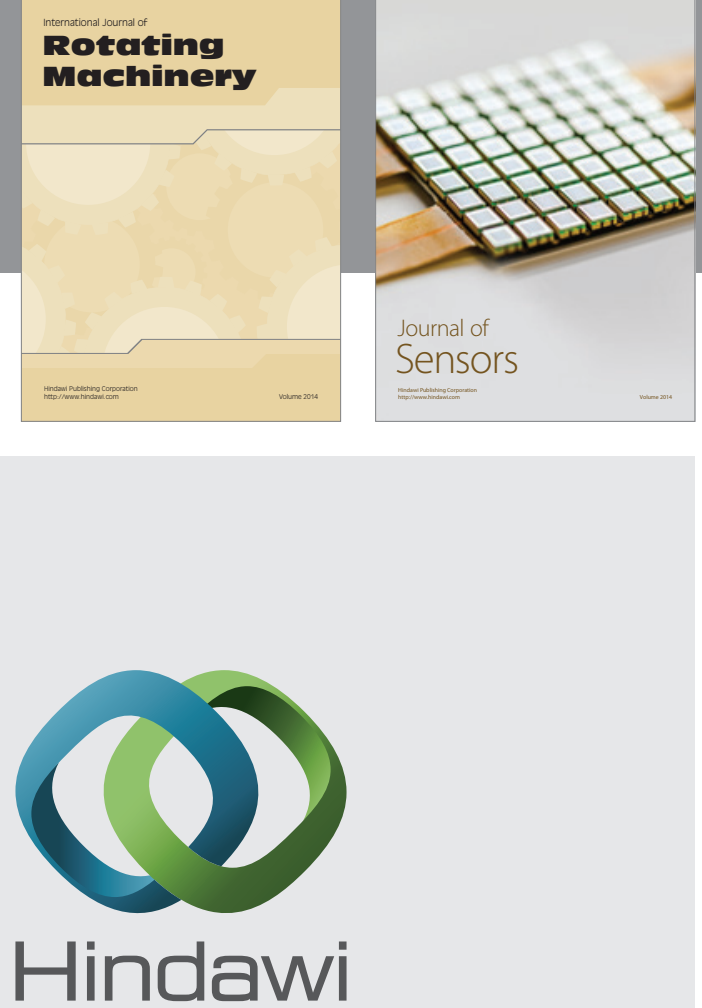

Submit your manuscripts at http://www.hindawi.com
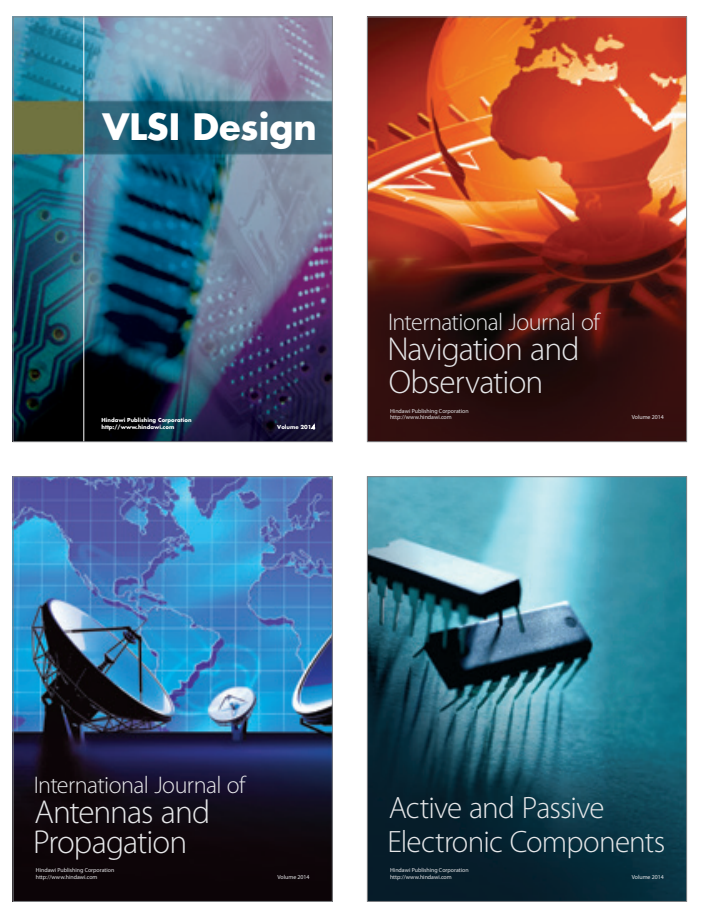
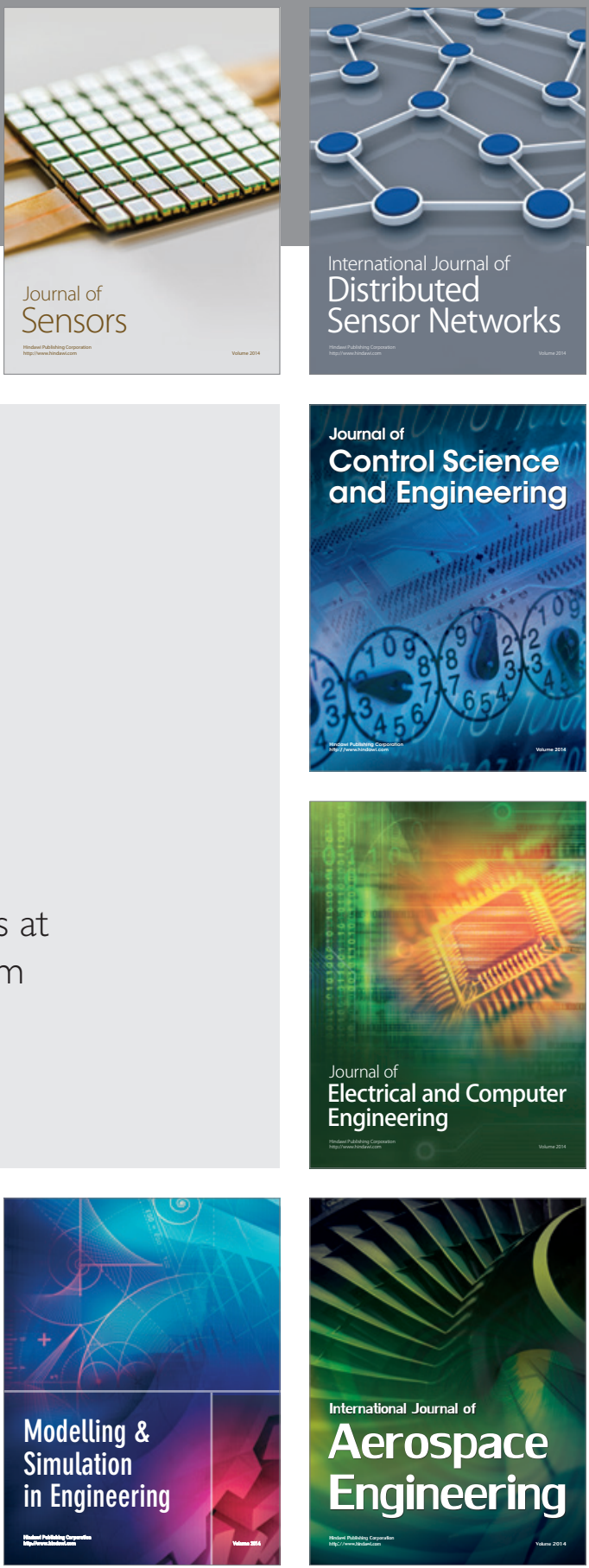

Journal of

Control Science

and Engineering
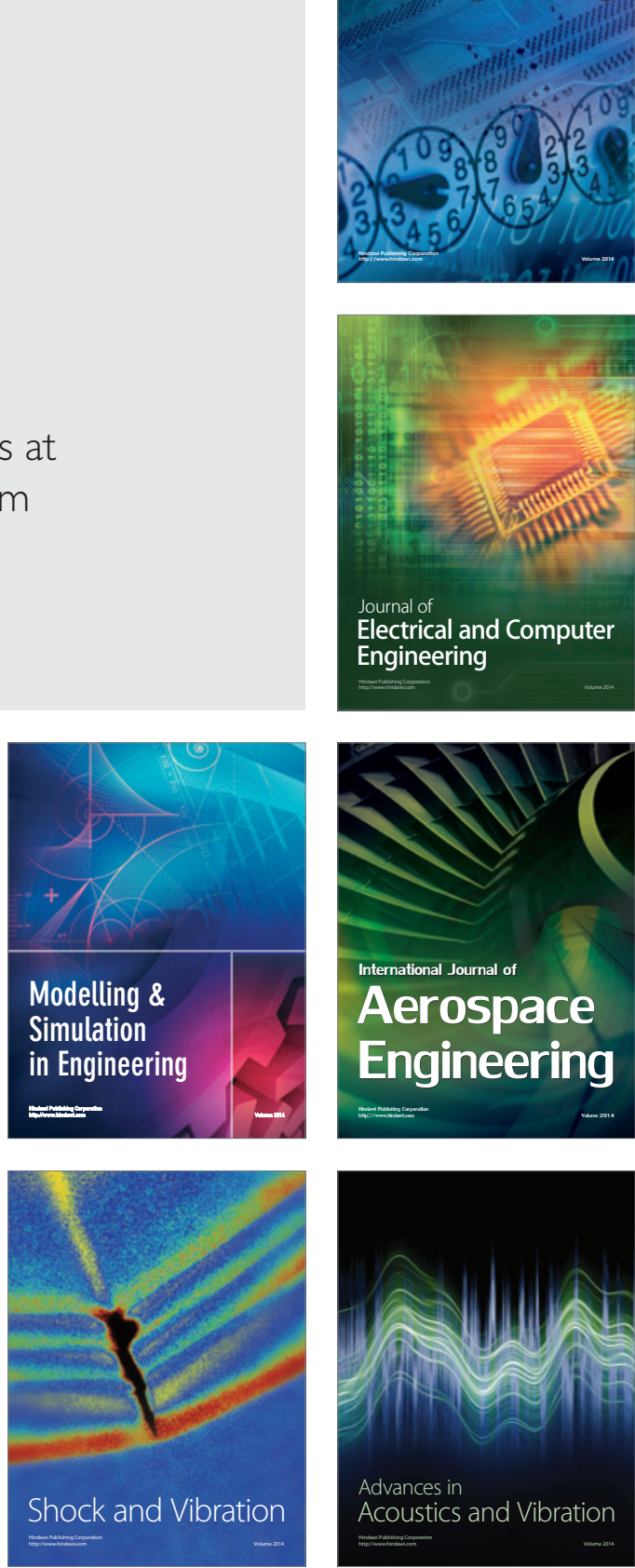